\title{
Systolic heart failure: should we care about electrical, mechanical or electromechanical dyssynchrony?
}

\section{Lorella Gianfranchi}

Division of Cardiology, SS Annunziata Hospital, Via Vicini 2 Cento (Fe) Italy m Tel.: +39051 6838251 - Fax: +39051 6838471 = l.gianfranchi@ausl.fe.it.

Evaluation of: Cho GY, Kim HK, Kim YJ et al.: Electrical and mechanical dyssynchrony for prediction of cardiac events in patients with systolic heart failure. Heart (2009) (Epub ahead of print). Prolonged QRS duration is electrocardiographic evidence of electrical dyssynchrony and is associated with a higher incidence of cardiac events and mortality. The study by Cho et al. (167 patients were followed up for 33.4 19.9 months) shows that mechanical dyssynchrony has an additional value over QRS duration in predicting cardiac events in patients with systolic heart failure. The dyssynchrony was assessed through tissue Doppler imaging - a temporal difference between the septal to lateral wall (Ts-1) of $65 \mathrm{~ms}$ or more defined the mechanical dyssynchrony. In multivariate Cox proportional hazard analysis, both QRS duration (hazard ratio (HR): 1.85; $p=0.032$ ) and Ts-1 (HR: 2.35; $p=0.002$ ) were independent predictors of cardiac events. Those with both electrical and mechanical dyssynchrony had a HR of 3.98 (95\% Cl: 2.02-7.86; $\mathrm{p}<0.001$ ) when compared with those with a normal QRS duration and absence of mechanical dyssynchrony. Combining the information provided by QRS duration with mechanical dyssynchrony through Ts-1 is clinically relevant to stratifying the risk of hospitalization or death in systolic heart failure patients. However, these data do not support the use of mechanical dyssynchrony to select patients for cardiac resynchronization therapy, nor to predict response to this therapy. This was not assessed by the study.

The paper by Cho et al. evaluates the independent role of electrical and mechanical dyssynchrony for predicting cardiac events in patients with systolic heart failure (HF) [1]. The prognostic value of prolonged QRS duration as a marker of ventricular dyssynchrony has been known for several years [2].

\section{Methods}

The study by Cho et al. is a single center, prospective, observational study. Patients with New York Heart Association (NYHA) functional class III or IV who were hospitalized for HF with a sinus rhythm and left ventricle (LV) ejection fraction of $35 \%$ or less, were consecutively enrolled. A total of 167 patients were included in the study.

Patients were treated with $\beta$-blockers or angiotensin-converting enzyme inhibitors (or angiotensin-receptor blocker) and diuretics at the time of discharge.

The time to peak systolic velocity from the $\mathrm{R}$ wave on a QRS complex during the ejection period was measured. LV dyssynchrony was derived from the time difference between the septum and lateral wall (regarding the time to peak systolic velocity in tissue Doppler imaging [Ts-1]). Patients were divided into four groups; group I: QRS duration of less than $120 \mathrm{~ms}$ and Ts-l of less than $65 \mathrm{~ms}, \mathrm{n}=71$; group II: QRS duration of $120 \mathrm{~ms}$ or more and Ts-l of less than $65 \mathrm{~ms}, \mathrm{n}=25$; group III: QRS duration of less than $120 \mathrm{~ms}$ and Ts-1 of $65 \mathrm{~ms}$ or more, $\mathrm{n}=44$; group IV: QRS duration of 120 $\mathrm{ms}$ or more and Ts-1 of $65 \mathrm{~ms}$ or more, $\mathrm{n}=27$. Clinical events included hospitalization for HF and cardiac death.

\section{Results}

In univariate analysis, older age, ischemic etiology, longer QRS duration, no $\beta$-blocker use and longer Ts-l were associated with cardiac events. In multivariate Cox proportional hazard analysis, both QRS duration (hazard ratio [HR]: 1.85; $\mathrm{p}=0.032$ ) and Ts-l (HR: 2.35; $\mathrm{p}=0.002)$ were independent predictors of cardiac events. Those with both electrical and mechanical dyssynchrony had a HR of 3.98 (95\% CI: 2.02-7.86; $\mathrm{p}<0.001$ ) when compared with those with normal QRS duration and an absence of mechanical dyssynchrony.
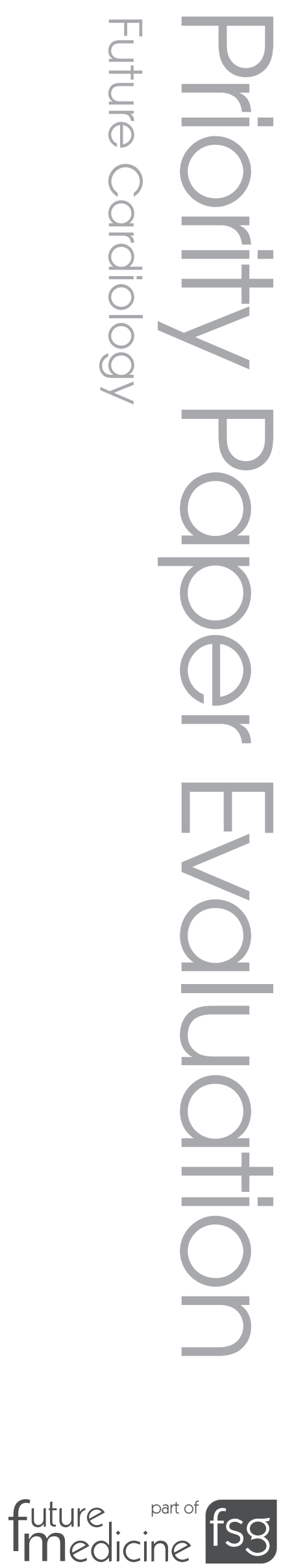
Cardiac events during follow-up were 23.9\% (Kaplan-Meier estimate; 17 events in 71 patients) in group I, $40.0 \%$ (ten out of 25 ) in group II, $59.1 \%$ (26 out of 44) in group III, and $63.0 \%$ (17 out of 27 ) in group IV ( $p<0.001)$. The mean event-free survival time decreased as QRS duration and Ts-l increased.

\section{Significance}

The major finding of this study was that mechanical dyssynchrony has an additional value over QRS duration in predicting cardiac events in patients with advanced HF. Thus, not only QRS duration, but also mechanical dyssynchrony is an independent and clinically relevant factor influencing a patient's outcome. We agree with this finding.

However, we do not agree with Cho et al. when they deduce that the results are also relevant for cardiac resynchronization therapy (CRT) [3]. This study does not show that mechanical dyssynchrony is a good parameter for selecting patients, nor that it can be corrected by the CRT.

We should not confuse predictors of outcome with parameters for selecting CRT patients.

Contrasting data have been published regarding the effectiveness of CRT in patients with mechanical dyssynchrony and narrow QRS [4,5]. In patients with narrow QRS, the possible presence of mechanical dyssynchrony may not be due to impairment of the electrical conduction system. This may be key to understanding the unsatisfactory results. On the contrary, CRT can correct the mechanical dyssynchrony caused by electrical dyssynchrony owing to left bundle branch block (LBBB) [6,7].

This rationale is also confirmed by the technique of pacing the left ventricular wall in synchrony with the spontaneous activation of the septum through the right bundle branch in patients with sinus rhythm and LBBB [8]. The resulting depolarization of the heart is the so-called fusion between the spontaneous activation of the septum and the pacing LV. This technique restores the electrical and mechanical timing of left ventricular-free wall activation, which is impaired by $\operatorname{LBBB}[9,10]$.

\section{Future perspective}

Mechanical dyssynchrony through echocardiographic assessment, combined with QRS duration, is clinically relevant to stratify the risk of cardiac events in systolic HF patients.

\section{Financial \& competing interests disclosure}

The author has no relevant affiliations or financial involvement with any organization or entity with a financial interest in or financial conflict with the subject matter or materials discussed in the manuscript. This includes employment, consultancies, honoraria, stock ownership or options, expert testimony, grants or patents received or pending, or royalties.

No writing assistance was utilized in the production of this manuscript.

\section{Executive summary}

\section{Background}

- Mechanical dyssynchrony may add valuable information for stratifying the risk of cardiac events in systolic heart failure patients.

\section{Methods}

- The study enrolled 167 patients with systolic heart failure, who were divided into four groups group I: QRS duration of less than 120 ms and Ts- 1 of less than $65 \mathrm{~ms}, \mathrm{n}=71$; group II: QRS duration of 120 ms or more and Ts- 1 of less than $65 \mathrm{~ms}, \mathrm{n}=25$; group III: QRS duration of less than 120 ms and Ts-1 of 65 ms or more, $n=44$; group IV: QRS duration of 120 ms or more and Ts-1 of 65 ms or more, $n=27$. Clinical events included hospitalization for worsening of heart failure and cardiac death.

\section{Results}

- Cardiac events during follow-up were 23.9\% (Kaplan-Meier estimate; 17 events in 71 patients) in group I, 40.0\% (ten out of 25) in group II, 59.1\% (26 out of 44) in group III and 63.0\% (17 out of $27)$ in group IV $(p<0.001)$. The mean event-free survival time decreased as QRS duration and Ts-1 increased.

\section{Significance}

- Not only QRS duration, but also mechanical dyssynchrony, is an independent factor that influences patients' outcome.

\section{Future perspective}

- Mechanical dyssynchrony through echocardiographic assessment, combined with QRS duration, is clinically relevant to stratify the risk of cardiac events in systolic heart failure patients. 


\section{Bibliography}

Papers of special note have been highlighted as:

-" of considerable interest

1. Cho GY, Kim HK, Kim YJ et al.: Electrical and mechanical dyssynchrony for prediction of cardiac events in patients with systolic heart failure. Heart (2009) (Epub ahead of print).

2. Iuliano S, Fisher SG, Karasik PE et al:: QRS duration and mortality in patients with congestive heart failure. Am. Heart J. 143, 1085-1091 (2002).

3. Chung ES, Leon AR, Tavazzi L et al.: Results of the Predictors of Response to CRT (PROSPECT) trial. Circulation 117, 2608-2616 (2008).

-. Multicenter clinical trial that evaluated the ability of several echocardiographic measures of mechanical dyssynchrony to predict response to cardiac resynchronization therapy (CRT) - no single echocardiographic measure of dyssynchrony may be recommended to improve patient selection for CRT beyond current guidelines.
4. Bleeker GB, Holman ER, Steendijk P et al: Cardiac resynchronization therapy in patients with a narrow QRS complex. J. Am. Coll. Cardiol. 48, 2243-2250 (2006).

5. Beshai JF, Grimm RA, Nagueh SF et al.; RethinQ Study Investigators: cardiacresynchronization therapy in heart failure with narrow QRS complexes. N. Engl. J. Med. 357, 2461-2467 (2007).

- Prospective, randomized trial providing evidence that patients with heart failure and narrow $Q R S$ intervals may not benefit from CRT.

6. Liu L, Tockman B, Girouard S et al.: Left ventricular resynchronization therapy in a canine model of left bundle branch block. Am. J. Physiol. Heart Circ. Physiol. 282, 2238-2244 (2002).

7. Ozdemir K, Altunkeser BB, Korkut B et al.: Effect of left bundle branch block on systolic and diastolic function of left ventricle in heart failure. Angiology 55, 63-71 (2004).

-. Left bundle branch block causes diastolic function impairment in normal subjects that is similar to that of patients with heart failure, and it also increases impairment of systolic function in patients with heart failure.

8. Gianfranchi L, Bettiol K, Sassone B et al.: Fusion beat in patients with heart failure treated with left ventricular pacing: may ECG morphology relate to mechanical synchrony? A pilot study. Cardiovasc. Ultrasound 6, 1-9 (2008).

9. Auricchio A: Pacing the left ventricle: does underlying rhythm matter? J. Am. Coll. Cardiol. 43, 239-240 (2004).

10. Verbeek XA, Vernooy K, Peschar M et al.: Intra-ventricular resynchronization for optimal left ventricular function during pacing in experimental left bundle branch block. J. Am. Coll. Cardiol. 42, 558-567 (2003).

\section{Affiliation}

- Lorella Gianfranchi

Division of Cardiology, SS Annunziata Hospital, Via Vicini 2 Cento (Fe), Italy Tel.: +390516838251

Fax: +390516838471

l.gianfranchi@ausl.fe.it 